\title{
Knowledge Management Systems in the Public Sector: Critical Issues
}

\author{
Stamatios A. Theocharis and George A. Tsihrintzis
}

\begin{abstract}
The transition from traditional government to e-government and from there to open government is connected with the burgeoning data and the appearance of open public data. It is significant to re-engineer solutions in the field of knowledge recovery and management, taking into account the semantic dimension of the data. In this paper, we present the concepts of knowledge, knowledge management and knowledge management systems. We also propose relevant architecture and present conclusions from the development of the ontology of e-government in Protégé.
\end{abstract}

Index Terms-Knowledge management, knowledge acquisition, knowledge coding, e-government, Protégé, OWL ontologies.

\section{INTRODUCTION}

In recent years internationally, there has been a clear need for more effective and efficient national administrative systems to promote the economic development of nations. This is why there has been a lot of discussion made about the change of public administration and in particular the implementation of an integrated plan for administrative reform throughout the public sector. E-government is being indicated as a vehicle for this reform. The application requires not only the implementation of infrastructure and communications technology and the interconnection of information systems, but also ensuring interoperability of information systems and processes. Institutional and organizational changes in the public sector that will enable public bodies to meet the needs of the new digital era, are also considered necessary.

The further promotion of e-government includes collective effort, exchange of experiences on best practices both at national and European level in order to disseminate secluded experience and knowledge. The environment in which public administration operates is characterized by production and distribution of vast size information resulting from the processing of an equally large dataset. The whole situation is exacerbated when employees leaving the service and do not leave behind them the valuable knowledge gained during the years of of service. Even in the case where organizational knowledge is "somewhere" inside the body, the recovery is extremely difficult and time consuming task. The result is obvious: waste of time, low service quality, reduced efficiency, and poor image to the outside.

Key success factor of the overall system is a fully

Manuscript received September 9, 2014; revised November 10, 2014.

The authors are with the Department of Informatics, University of Piraeus, Greece (e-mail: stheohar@unipi.gr, geoatsi@unipi.gr). functional, efficient and effective back-office. For the improvement or development of existing systems, emphasis should be given, apart from institutional reforms, innovation, cutting-edge technologies and knowledge management systems. The challenge in the near future is the creation of mechanisms that will discover, draw, exploit and distribute the knowledge of each individual employee in order to increase productivity and efficiency of services.

The present paper is an attempt to identify issues related to the concept of knowledge, consolidation and knowledge management systems, as initial steps of the procedure of developing a management mechanism in the public sector.

In particular, the work is organized as follows: in the second section we present a summary of the previous work presented in [1] and relates the concepts of knowledge, its creation and its codification, and also its correlation with application examples in the public sector. The third section presents issues related to knowledge management and the fourth section presents issues on the development of knowledge management systems. The fifth section presents issues about the ontology implementation in Protégé and in the sixth and last section there are conclusions and future work.

\section{PREVIOUS WORK}

\section{A. The Concept of Knowledge}

The concept of knowledge in various fields of human activity has its roots in antiquity, as revealed by the works of the Greek philosophers. Knowledge stems from people through their experiences and ideas and subject to the possession skills that support the perception of reality and the existence of an objective reality. In Western philosophy it was considered that Knowledge as a true creation with physical entity can be reproduced and therefore suitably used, by means of technology. Initially, during the development of relevant tools with the help of IT, knowledge is treated as an object while it is now considered as the reason for the partial failure of these. Instead, it is now accepted that a highly active participation of people in provisioning, updating and management of knowledge information systems is essential [2].

For a proper understanding and application of knowledge management it is necessary to specify the concepts of data, information and knowledge, which are closely linked and interdependent [3]. Through a practical example of the Greek public administration there are empirical definitions of these concepts. The Ministry of Interior in the context of the implementation of the "Politeia" project, relating to the civil 
service enables telephone request for specific services. The knowledge that can be generated from the initial pilot operation of the service is associated with the following:

- The data can include elements, measurable or qualitative, which are recorded on a daily basis, such as the allocation of telephone applications (measurable) per category of certificate (quality), which will become meaningful if placed in a specific structure and organized through specific framework.

- Information obtained when the data collected by the employees is processed with some application and the characteristics of supervisory applications are presented.

- The knowledge is generated during the interpretation and discussion between the employees after a certain operating period of the system.

- The improvement of the services provided through a revision of the procedures is associated with the wisdom acquired by the employees who designed this program.

\section{B. Creation of Knowledge}

The creation of knowledge regarded as a constantly repeated process starts from one or more people by sharing new ideas, events or problems. This is followed by individual or group learning, information acquisition, evaluation and finally, building of organizational knowledge [4].

In the case of public sector organizations, knowledge extends to all the procedures to be followed in order to produce public services to the public. The procedures may involve both communication between departments at the level of back-office and the interaction of the public with public bodies. The implicit knowledge can be found in employees with expertise in specific issues and recovered primarily through informal situations, work culture or informal groups of employees arising in the workplace. It's the kind of knowledge that is difficult to standardize and to accept treatment. Explicit knowledge characterizes the entire operation of the public sector and is based on statutory texts. The difficulty in management is associated with a huge number of relevant provisions, legislation, circulars, directives, etc. but overlaps or conflicts between them.

During the process of creating and transforming knowledge new concepts or ideas are developed, some of which in the environment of public organizations will require sanction at a higher hierarchical level to provide knowledge that will be distributed to all official bodies. Other ideas or concepts derived from everyday work practice or may be the subject of discussion and sharing among employees at a lower hierarchical level. For all these we need specially designed operating environments that facilitate the communication of ideas between all the units or employees. Building organizational knowledge (tacit knowledge elements based on the experience of workers and to concrete data and information which can be stored in the archives of the organization / institution) relies heavily on the internalization methodology of individual or collective knowledge in the knowledge base of organization

\section{Encoding of Knowledge}

Knowledge can be shared and used by all employees of an organization to the extent that can be easily or harder to codify. The experiences of the officials which cannot be identified are extremely difficult to become knowledge of the agency. Instead, the knowledge that can be presented in a tangible / explicit form may be distributed and receive processing at low cost and in short time. The coding allows knowledge to be better understood, maintained and improved and become part of the organizational memory [5]. The codification of knowledge as a process is not always possible and depends on the type of knowledge. The codification of explicit knowledge can be achieved through various techniques such as cognitive mapping, decision trees, taxonomies and knowledge analysis of tasks, which have been analyzed in previous work [1].

\section{KNOWLEDGE MANAGEMENT}

\section{A. The Concept "Knowledge Management"}

Knowledge management can be used by almost all government ministries and organizations, although considered to have more visible results in relatively large companies and companies in technology-intensive or knowledge [6]. With knowledge management is possible to address problems of public administration such as:

- When there is a need for a specific expertise or skill and none of the existing staff seems to have such knowledge.

- When a solution to a problem requires some experience but the person who has this experience is missing.

- When the matching of the person (meaning its abilities) and the job does not work efficiently while optimal matching between position and specific, objective abilities.

- When an organization decides to apply policies of staff training, but training needs, which reflect specific knowledge gaps are not known and have not been documented.

- When there is knowledge to certain individuals, however, either is not used or not effectively diffused the Organization for a variety of reasons

Although the application of knowledge management varies greatly between organizations in the public and private sector, generally the value of knowledge management can be found in the following areas:

- Decision making. With knowledge management can achieve better and faster decisions. This is because the experience of the organization is recorded and this helps both to avoid mistakes and discover and use the best solutions to similar issues saving time.

- Autonomy resources. The involved employees become able to access and use the knowledge of their peers while enhancing their responsibility and control of their performance.

- Learning. More rapid learning by compressing the length of the learning curve for any new object assigned to employees.

Although there are many business and organizational problems related to knowledge management, however oddly enough occur during the daily routine of organizations in both the private and public sectors:

- Employees have knowledge gained before entering the service or can be trained during their work (e.g. seminars, refresher courses, etc.) but are not allowed to use them for 
various reasons (e.g. political considerations, failure in matching job and person etc.).

- Employees during the exercise of their duties acquire knowledge but most do not transmit it to the other employees involved.

- Often there is the right person who can solve the problem, but few know how to locate him.

- Every organization wants to have the best employees, but when they acquire them and train them properly, for various reasons those either change subject or even organization.

- Employees while asked to solve problems and ask and seek the knowledge of their colleagues but in many cases they keep their knowledge to themselves.

- Employees must document their deliverables fully, but often do not have easy access to the relevant files.

\section{B. Critical Issues for the Implementation of Knowledge Management in the Public Sector}

Public bodies in order to have maximum results should align knowledge management with key strategic objectives. These targets are either known from the existing business plan of the entity or by conducting a SWOT analysis, from which we derive the corresponding priorities / objectives. This is crucial because in most cases, the results of knowledge management are not evident in terms of the costs or other quantitative characteristics and in order to ensure full commitment of management, management knowledge team should present as soon as possible the first results.

\section{1) Diagnosis of the organization's knowledge}

We must identify knowledge gaps, future requirements in knowledge and the specific knowledge characteristics of each institution. The well defined strategic objectives, providing a suitable framework for analyzing the gaps in current knowledge and future needs of the body of knowledge. Knowledge gaps can arise by the absence of people (special expertise or expert) or lack of technology or both. Knowledge gaps should be filled by various techniques, such as internal training, hiring new staff acquisition of expertise, production of new knowledge, etc. The potential knowledge gaps could be identified by problems that have been reported in the past, and after discussion with the heads of the organizations' units and the entire staff. This process is also likely to identify surplus of knowledge in some units. This process can be done by using appropriate Diagnostics Knowledge Management Tools. These tools can provide the user with a detailed methodology on how to identify all areas (process identifier), which should be explored and further improved. In this way it is possible to identify some characteristics of the organization regarding knowledge management and remove all the obstacles.

\section{2) Implementation of a policy changing culture}

In today's competitive work environment many employees do not feel safe on the job position and usually conceal their knowledge, which could be used to improve the overall efficiency of the department or the organization. From another perspective, employees may hide critical knowledge, because they believe that if they share it with others they will lose their personal knowledge advantage. This is probably the biggest hurdle for any knowledge management activity. Employees must feel free to share ideas, make mistakes or ask other employees if they do not know something.

People in their workplace should not be afraid to discuss or read a book even while working, in order to gain new awareness. All these minor, but still very critical, daily activities can help an organization to build a culture of knowledge management. It is equally important that the hierarchically highest executives communicate to the lower ones the strategic goals of the organization in order that the necessity of any changes will be clearly known.

\section{3) Using appropriate technologies}

For Knowledge Management, the role of innovative IT systems and the Internet next generation is catalytic. However, it is useful to note that their own infrastructure and applications are not sufficient for optimal results. Examples of some of the technologies that assist in knowledge management:

4) Tools for the identification and organizing of knowledge

These include:

- Search applications based on a word or an expression, such applications are provided so far by various private providers, but they do not focus on the semantic analysis of terms. However for the modern needs of the public sector it is essential to use search techniques that give solution to the problem of semantic correlation between search terms.

- Tools such as intelligent agents could offer solutions to the issue of filtering the information requested or applications depending on the needs of the employees.

- Pattern recognition techniques and text comparison techniques will facilitate to the clustering, categorization and classification of public government documents based on similarities in the terminology they use.

- Data warehouses, in the sense of interconnected databases on all public sector bodies

- Smart Tools tracking such information or document-based question answering undirected. Such tools can be used for data mining.

- Application of a knowledge mapping tool. The knowledge mapping is a process that aims to link knowledge to describe the people who possess it. In most cases it is difficult to map the knowledge itself and is easier and logical to create links between a brief description of knowledge and its holders. However, the knowledge in an organization exists in many forms and refers to various subject areas (administration, production, technical issues, etc.). Therefore, the knowledge in order be to described and fully understood by all, has to be expressed in a familiar and easy language (codified knowledge). There are many types of coding and the most appropriate should include knowledge of the specific characteristics of the company.

\section{5) Collaboration tools}

Herein are meant those technologies that support continuous communication and sharing of knowledge, ideas and solutions from all the officials concerned. In particular 
characteristic functions must be:

- The real-time communication via messages between agents are logged into the system each time

- The asynchronous communication, to the logic of e-mail

- The ability to form communities with common objects employment and knowledge level.

\section{6) Analysis and visualization tools}

Herein means tools that are able to interpret the input information but also to transform input data into detailed graphics. In this area, the virtual environment will allow users a more efficient collaboration between remote partners compared to traditional techniques.

\section{7) Decision support systems}

Herein means systems interacting through electronic means, in order to assist users in problem solving and decision making. Such systems take into account the input data and use specific models to replace the administrative crisis. The development of intelligent decision support systems that learn from the usage history of each user and the organization would have significant advantages over existing systems that have been developed.

\section{Knowledge Management Procedures}

Knowledge management focuses on capturing the expertise of organizations / institutions and individuals, as well as the allocation and application of this expertise to maximize benefits and profits derived from the assets of knowledge. Knowledge management help to transfer the appropriate knowledge to the right people at the right time, giving them the power to make the right decisions. Still, involves the identification and analysis of the existing and the required knowledge, and the subsequent planning and control of operations required for the development of knowledge assets and the fulfillment of the objectives of the organization / institution.

In order for the knowledge to be transformed into a valuable corporate asset, knowledge, experience and expertise should be standardized, distributed, to divide and implemented [7]. Knowledge Management is an integral part of any strategy that utilizes the expertise to create a sustainable competitive advantage in today's business environment. Many researchers formulated templates for knowledge management comprising from 2 to 8 or more different processes among which the following [8], showing in Fig. 1:

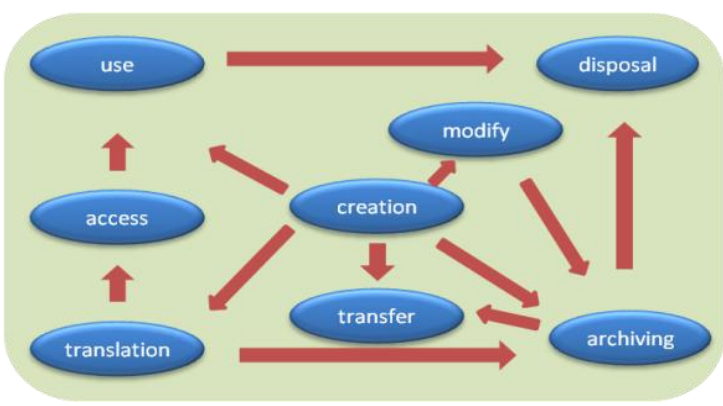

Fig. 1. Processes of knowledge management.

- Creation or acquisition. Knowledge either created or collected by knowledge workers.

- Modify. Knowledge modified, adapted or combined to meet immediate and future needs.

- Use. This knowledge is used for a particular useful purpose.

- Archiving. The knowledge is stored in a specific format (format) which will be maintained in perpetuity and will be accessible and useful for future use by knowledge workers of the organization / institution (encoding).

- Transfer. Knowledge can be transferred or communicated from one person or place to another.

- Translation / redefinition of purpose. This knowledge is translated from its original form to a new form which is most suitable to achieve a new goal.

- Access user. Knowledge is accessible to knowledge workers depending on the position of responsibility held by the organization.

- Disposal. It is important to recognize which information / knowledge one should keep and which one to delete.

We note that in the context of knowledge management practice, these processes can be grouped into more general processes that are more appropriate for business processes implemented by the organization / institution.

\section{KNOWLEDGE MANAGEMENT SYSTEMS}

With the concept of "system" knowledge management refers to a collection of technological infrastructures which are connected with each other and with their environment with specific interfaces [9], [10]. People, content, technology (software \& hardware) and their interfaces are the interdependent and inseparable components of such a system. People who possess knowledge (explicit or implicit) offer content-knowledge, and the transfer and diffusion of the whole organism is through technology. This combination provides effective and efficient management of an organization's core knowledge (see Fig. 2).

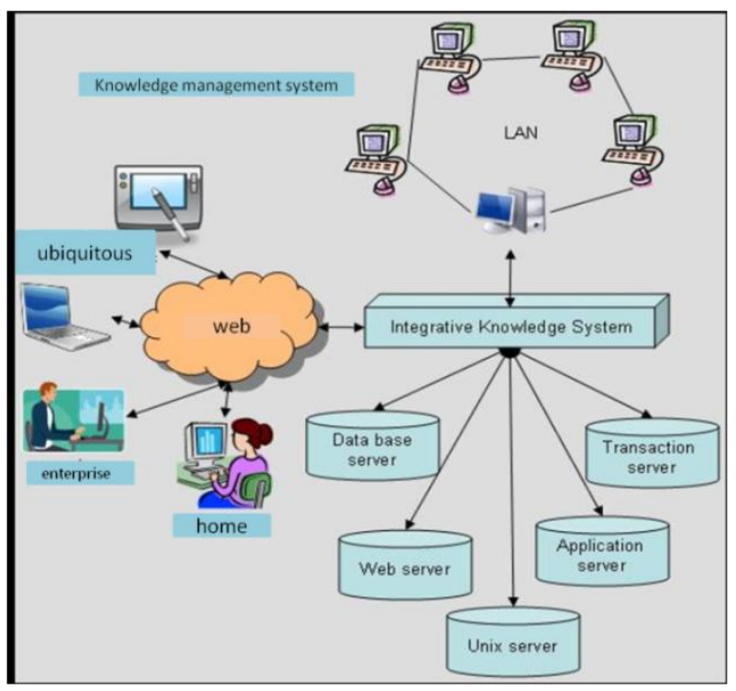

Fig. 2. The structure of a knowledge management system.

From a technological point of view, an interesting architecture of such a system is shown in the following Fig. 3 the 7-level subsystems.

\section{A. First Layer}

The first layer of the proposed architecture is actually a unique subsystem-level of interaction between user and 
machine. It can have the form of a browser or a machine wiki. The aim of this module is to support the flow of both the explicit and the tacit knowledge and information to and from the man and the machine. This transfer can be made more efficient and effective by using a personalized system that will use appropriate teaching aids, such as graphs, tables, charts and text. Thus, the explicit and tacit knowledge will be presented in a consistent, navigable, friendly and consistent manner regardless of the source of the turnout.

Since this layer acts as an interface between the user and the system, the question of design is extremely important and various features must be taken into account like:

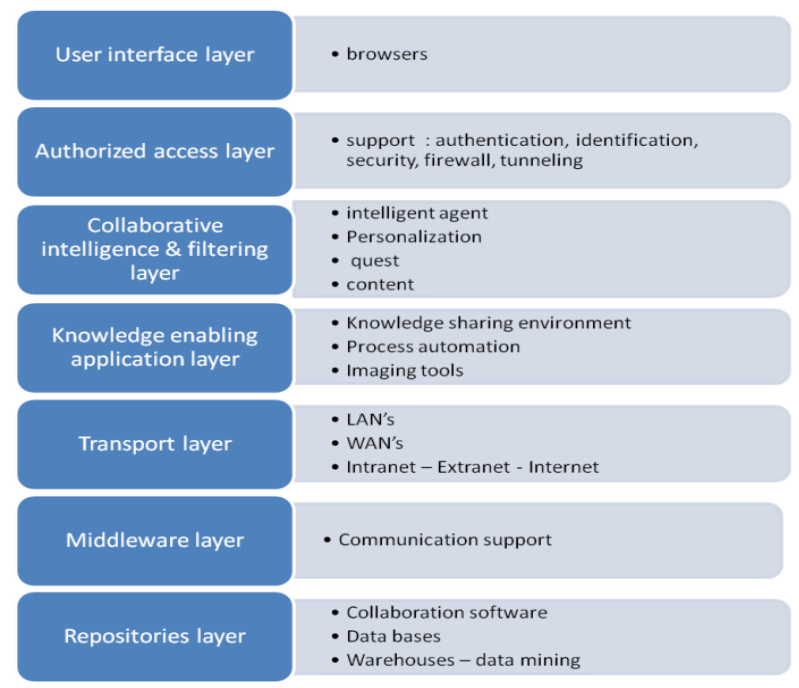

Fig. 3. The 7-level subsystems.

- Consistency. As with any other software we must use menus, buttons and icons with the same appearance and functionality

- Relevance. The information provided must be strictly relevant to the user's requirements.

- Optical clarity to the extent of saving space in the output means of information

- Optical clarity to the extent of saving space in the output means of information

- Easy navigation between pages and related files

- Maximum usability. The usability of the various menus and icons, the speed of access to files and websites are among the basic users require.

At this level, we must address the issue of managing tacit knowledge. The tacit knowledge embodied in the intellect of experts or employees engaged for a long time to a specific object, it is difficult to codify and share with existing systems. It is therefore a major challenge to develop a subsystem that supports the automated recording and mapping of tacit knowledge of government officials.

\section{B. Authorized Access Layer}

Here is where the protection of the system and controlled access to knowledge found in computer systems of the body are ensured. It is extremely important because such systems enable remote access via internet or via intranet, so they are particularly vulnerable to malicious attacks. A modern access layer focuses on safety, on the use of protocols such as passwords, on certification to ensure that they are authorized users and software tools such as firewalls that prevent the exit of certain sensitive personal data or the entrance of information that can destroy the records of the organization.

\section{Collaborative Intelligence and Filtering Layer}

The third layer provides in a personalized way the stored information. Designed to reduce the seek time of information and the relevance to the actual knowledge searched in conjunction with the user profile. This feature is provided via a search engine such as a browser or client / server network of the company in conjunction with the first level of the architecture. In this field, intelligent agents can give meaningful solution. Intelligent agents are active tools that can understand, analyze (learn from past experiences) and take appropriate action to help solve a problem.

\section{Knowledge-Enabling Application Layer}

Most of the applications developed at this level, aim at knowledge management and learning by providing users better ways to perform their work. May include discussion databases, automation tools for often repetitive tasks, phonebooks decision support, tools, visualization tools or other specialized applications. The ultimate goal is to provide users with knowledge about specific tasks that they perform.

\section{E. Transport Layer}

This level, includes local area networks - LANs to wide area networks - WANs, the intranet and extranet as well as the Internet. The basic technical characteristics which must be considered are the interface speeds, bandwidth (bandwidth), the tools for managing web traffic / load.

\section{F. Middleware Layer}

Here there is the interoperability of different systems. During the system design we must take into account the way the system Ais communicate with existing systems and applications that have been developed on different platforms or run on different operating systems.

\section{G. The Repositories Layer}

This is the lowest layer of the architecture and DK represents the physical layer in which the points of data storage are located. Includes data warehouses, legacy applications, operational databases and custom applications to manage network traffic / congestion and ensure the integrity of the architecture Ais. Each storage location has the required structure for the type of knowledge that is stored.

\section{MODELING AND EXTRACTION OF KNOWLEDGE IN THE FIELD OF E-GOVERNMENT}

\section{A. Ontology Implementation}

For the extraction of knowledge in the field of e-government and open public data, we developed the ontology of e-government in the environment of Protégé 4.2. That ontology models the overall governance environment, structures and functions of public administration and the relationships between them. In this work, we extended the original model by adding new concepts (classes) and the introduction of indicative instances (individuals) in order to control the behavior and possibilities offered by the environment of Protégé. Part of the ontology is shown in Fig. 
5 below. The accuracy and consistency of the ontology were tested by means of the easoned HerniT provided by Protégé.

\section{B. The SPARQL Query Tool Protégé as a Means of Information Retrieval}

We used the SPARQL Query tab of the Protege, searching for information from the knowledge base created through the ontology, in the local environment of our computer. We are particularly interested in this tool, because SPARQL provides the ability to retrieve information by querying knowledge bases mainly oriented to RDF triples, but without excluding relational databases and datasets with semantics derived from spreadsheets. Full utilization of these questions in the Internet environment is achieved through SPARQL EndPoints on the general architecture of Fig. 4.

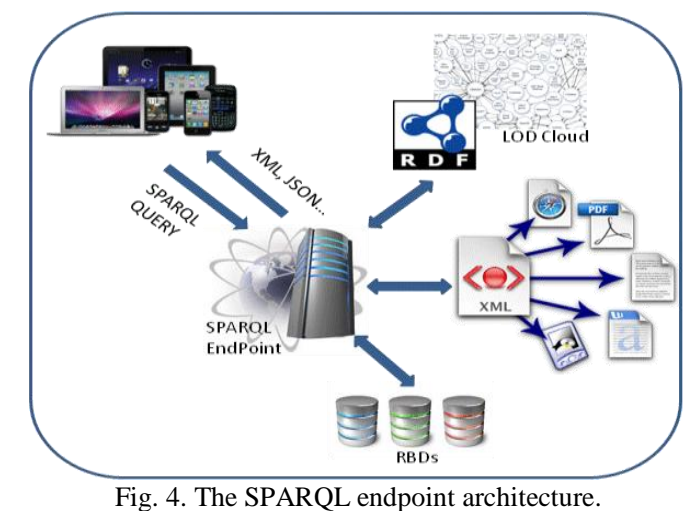

A SPARQL EndPoint is nothing but a web service through which a server can accept a SPARQL query from the client, then perform the appropriate search depending on the data set that is addressed by the client and finally return the results through the protocol HTTP [11]. However there are still problems to be solved such as the availability, maintenance and consistent performance of endpoints.

\section{Remarks by Implementing SPARQL Queries in Protégé}

Since the queries implementation to the knowledge base of the ontology using SPARQL Query Tab of Protégé, we note the following points as crucial for further implementation in the environment of a SPARQL endpoint.

- Particular care must be taken when modeling knowledge to be as compatible as possible with RDF triples.

- When defining concepts, initial declarations of classes and their properties are important in order to avoid inconsistencies and logical errors which do not appear in the original design. Although protégé provides the ability to control the accuracy and consistency of the ontology through the easoned, this is not useful in the performance of queries. This happens because queries are executed on the RDF triples created by the definition of ontology and not in subsequent relationships.

- When implementing a SPARQL EndPoint we must predict the recovery and presentation to the end user, of the properties associated with the concepts of ontologies. Although the basic concepts are usually known in advance to the end users, their properties and their relationships depend on the initial design and modeling of knowledge in accordance with the visual of the designer.

- The results of queries submitted to the SPARQL Query
Tab of Protégé, originally depend on declared properties rather than those deduced from the reasoners of Protégé.

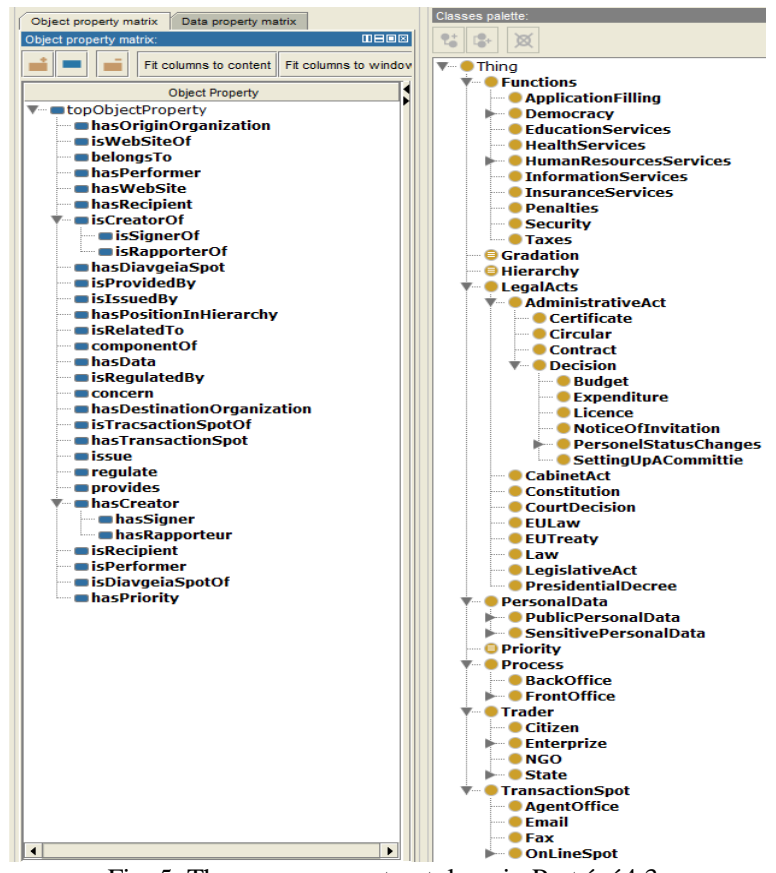

Fig. 5. The e-government ontology in Protégé 4.3.

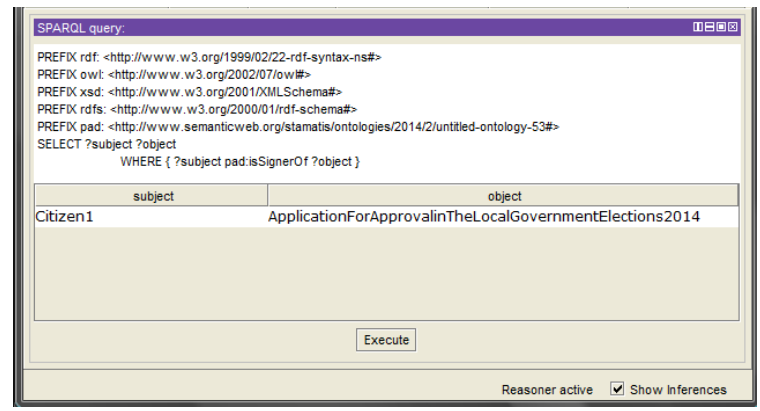

Fig. 6. SPARQL query example on e-government ontology in Protégé.

- A disadvantage we note for SPARQL Query Tab of Protégé is that it does not provide the ability to store queries for future use.

\section{CONCLUSIONS AND FUTURE WORK}

The optimal use of knowledge that can be produced from information maintained by the public sector is proving particularly crucial. This is because it is linked to 1) the efficiency of the public sector, 2) saving resources and 3) the adoption of innovative services. In the future, our interest will be focused on the automation of the production and the use of this knowledge. We will emphasize the development of knowledge-based applications to support back-office systems in the public sector. Also, our direct future interests include the development of a SPARQL EndPoint in order to exploit dynamically the open public data.

It is clear that when employees required to produce public services are supported by systems based on implicit knowledge, they can provide better public services.

\section{REFERENCES}

[1] S. Theocharis and G. Tsihrintzis, "Knowledge acquisition for the further development of e-government," JCKBSE 2012, pp. 286-297, 2012 . 
[2] Nonaka, "The knowledge creating company," Harvard Business Review, July-August 2007.

[3] J. B. Wang and H. C. Wang, "The research of e-government governance mode under knowledge management," in Proc. 4th International Conference on Wireless Communications, Networking and Mobile Computing, 2008, pp. 1-4.

[4] J. Paralic and T. Sabol, "Implementation of e-government using knowledge-based system," in Proc. 12th International Workshop on Database and Expert Systems Applications, 2001, pp. 364-368.

[5] M. A. Wimmer, "Implementing a knowledge portal for egovernment based on semantic modelling: The e-government intelligent portal," in Proc. 39th Annual Hawaii International Conference on System Sciences, vol. 4, 2006, p. 82b.

[6] Sarantis, Y. Charalabidis, and D. Askounis, "A goal oriented and knowledge based e-government project management platform," in Proc. 43rd Hawaii International Conference on System Sciences, 2010, pp. 1-13

[7] X. K. Yang, Y. Z. Wang, X. N. Zhang, and J. Jin, "Research on the classification of e-government information resources by categories-theme integrating," in Proc. 4th International Conference on Wireless Communications, Networking and Mobile Computing, 2008, pp. 1-4.
[8] Trainmor

[Online].

Available: http://www.trainmor-knowmore.eu/root.el.aspx

[9] P. Zhou, "An integrated architecture for effective knowledge e-government," in Proc. IEEE International Symposium on Knowledge Acquisition and Modeling Workshop, 2008, pp. 1125-1128.

[10] Y. Y. Dang, Y. C. Wang, and H. M .Wang, "The design and implement of e-government information system based on knowledge management," in Proc. 2011 International Conference on Mechatronic Science, Electric Engineering and Computer, 2011, pp. 2131-2134.

[11] SPARQL

[Online].

Available: http://semanticweb.org/wiki/SPARQL_endpoint

Stamatios A. Theocharis was born in Athens. He is a graduate of the Department of Mathematics, University of Ioannina-Greece and a post-graduate of the Department of Informatics, University of Piraeus-Greece. He is currently a $\mathrm{Ph}$. D candidate in the domain of e-government with his supervisor Professor George Tsihrintzis.

His current professional occupation concerns the object of the ministry of interior of Greece, open data, semantic web and the technologies that support it and the e-government technologies are included in his research interests. 